Ankita Rane* and Vadivel Sangili

\title{
Implementation of Improved Ship-Iceberg Classifier Using Deep Learning
}

https://doi.org/10.1515/jisys-2018-0271

Received June 25, 2018; previously published online July 24, 2019.

Abstract: The application of synthetic aperture radar (SAR) for ship and iceberg monitoring is important to carry out marine activities safely. The task of differentiating the two target classes, i.e. ship and iceberg, presents a challenge for operational scenarios. The dataset comprising SAR images of ship and iceberg poses a major challenge, as we are provided with a small number of labeled samples in the training set compared to a large number of unlabeled test samples. This paper proposes a semisupervised learning approach known as pseudolabeling to deal with the insufficient amount of training data. By adopting this approach, we make use of both labeled data (supervised learning) and unlabeled data (unsupervised learning) to build a robust convolutional neural network model that results in a superior binary classification performance of the proposed method.

Keywords: Binary classification, deep learning, neural networks, pseudolabeling, semisupervised learning.

2010 Mathematics Subject Classification: 68T45.

\section{Introduction}

Marine surveillance and circumstance awareness is very critical for monitoring and controlling piracy, smuggling, locating large-sized icebergs, and environmental hazards such as oil spills or pollution. In remote areas of the East Coast of Canada, drifting icebergs have possessed a great threat to mankind as well as marine activities by hampering navigation [6]. It has become increasingly difficult to monitor the movement of the icebergs even with the help of surveillance drones due to harsh climatic conditions in remote areas. This is where satellites come into use to monitor ships and icebergs despite the harsh weather conditions [8]. An international energy company, Statoil, has worked in collaboration with C-Core to come up with a computer vision-based surveillance system. To keep marine operations safe and effective, Statoil wants to get a crisp new point of view on how to use machine learning (ML) to build a more precise surveillance system to identify and detect large chunks of icebergs that are a potential threat; as a result, this dataset was made publicly available and is popularly known as the Ship-Iceberg Classifier Challenge [3].

One of the recent works on classifying ship-iceberg using predictive modeling was done by Jang et al., which uses several supervised learning algorithms such as random forest model to distinguish between ship and iceberg synthetic aperture radar (SAR) images. They further went on to train the convolutional neural network (CNN) on the available labeled train set and achieve a validation accuracy of $90 \%$ [3]. Another significant effort on ship-iceberg classification was by Bentes et al. They presented the use of CNN for ship-iceberg discrimination in high-resolution TerraSAR-X-images. Their dataset comprised 600 samples after data augmentation; the CNN was trained on $90 \%$ of the sample and tested on the remaining $10 \%$. The CNN model was compared to the support vector machine (SVM) model and the final results showed that the CNN model outperformed the SVM model due to better generalization ability [1]. A similar work was done by Jun et al. They trained the CNN on an available training set of 1604 images and achieved a validation accuracy of $88.33 \%$ [4].

\footnotetext{
*Corresponding author: Ankita Rane, Department of Computer Science, Birla Institute of Technology and Science (BITS) Pilani - Dubai Campus, Dubai, United Arab Emirates, e-mail: ankitarane24@gmail.com

Vadivel Sangili: Professor in Department of Computer Science, Birla Institute of Technology and Science (BITS) Pilani - Dubai Campus, Dubai, United Arab Emirates
} 
In contrast, our approach of semisupervised learning not only makes use of training samples for model learning but also incorporates the unlabeled test set data. Our CNN model being exposed to a large number of data samples is able to learn more features about the images, thus resulting in a superior learning performance.

Data scarcity is one of the main problems of ML and data mining tasks as insufficient data size is very often responsible for poor learning performances. Our work makes a significant contribution by proposing a solution to tackle the scarcity of labeled data for training purpose. This paper crafts a critical solution using unlabeled data to extract significant information for inferences. Our approach of pseudolabeling the unlabeled test set data to incorporate in the model training has improved the model robustness by creating a more precise decision boundary, ultimately resulting in the state-of-the-art performance by the prediction model. The experimental results revealed the effectiveness of our proposed approach when comparing the classification results to the $\mathrm{CNN}$ model without pseudolabeling.

\section{Data Extraction}

In this work, the dataset contains SAR images from the Sentinel-1 satellite housed $600 \mathrm{~km}$ over the Earth's surface. A total of 10,028 (test + train) satellite images are present in the dataset. Each image has a dimension of $75 \times 75$ pixel with two bands. This means that every band has 5625 positive floating values associated with it. The training set contains 1604 images that are labeled with the target variable (is_iceberg) and the remaining 8424 images belong to the test set that are unlabeled. Of the 1604 training samples, 753 are labeled as iceberg and 851 images are labeled as ship. Every training sample has an attribute, namely incidence angle (inc_angle), which gives us information about the angle at which the radar signals were incident on the object. The huge difference in the train and test datasets as well as a larger portion of the data being unlabeled poses a challenge in the process of effective classification. This has led us to follow the approach of semisupervised learning. The implementation of the code was entirely done using Spyder, which is a powerful development environment for Python language with advanced editing, testing, and numerical computing environment. The following are hardware specifications of the system are as follows:

System: ThinkStation-P500

Processor: Intel Xenon® CPU E5-1620 v3 @ 3.5 GHz $\times 8$

Graphics: Quadro K2200/PCle/SSE2

OS Type: 64-bit

Disk: $193.1 \mathrm{~GB}$

\section{Data Preprocessing}

Sentinel-1 satellite is at about $680 \mathrm{~km}$ above Earth, sending pulses of signals at a specific incidence angle and then recording it back. Those reflected signals are known as backscatter. The data we have been given is the backscatter coefficient that is the conventional form of backscatter coefficient given by the following equation:

$$
\sigma_{\circ}(d B)=\beta_{\circ}(d B)+10 \log _{10}[\sin (i p) / \sin (i c)]
$$

where ip is the angle of incidence for a particular pixel, ic is the incidence angle at the center of the image, and $\mathrm{k}$ is a constant. The Sentinel satellite is very similar to RISTSAT (an Indian remote sensing satellite) as it transmits pings in $\mathrm{H}$-polarization and not in V-polarization. $\mathrm{H}$ pings when incident on an object get scattered and return as a blend of $\mathrm{H}$ - and $\mathrm{V}$-polarizations. As Sentinel has only $\mathrm{H}$-transmitter, the return signals are of the forms $\mathrm{HH}$ and $\mathrm{HV}$ only.

Before we use the data for analysis to bring meaningful insights, we first preprocess it to remove unnecessary information present in it. We replace the rows with inclination angle as "na" with a zero. Each training sample has two bands (HH and HV) associated with it. We merge the two bands by averaging the two and 


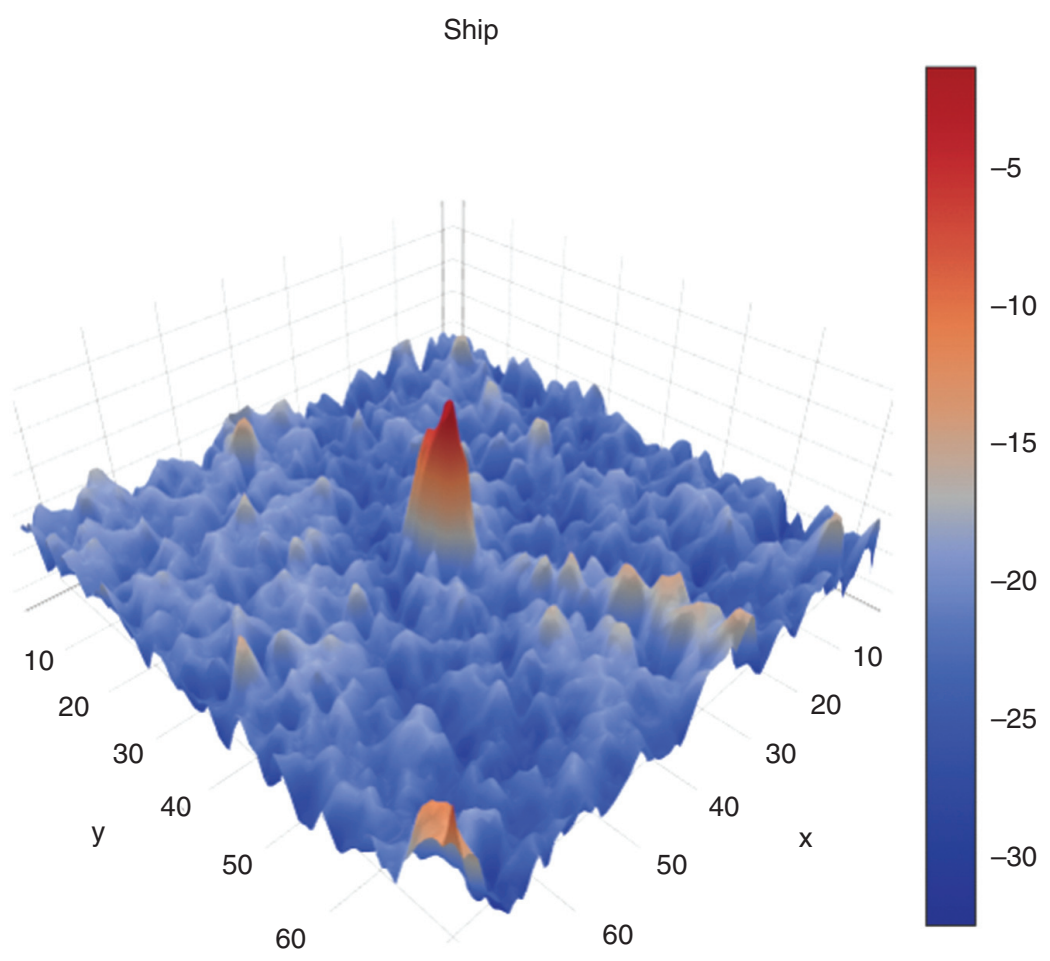

Export to plot.ly n

Figure 1: 3D Plot of a Ship.

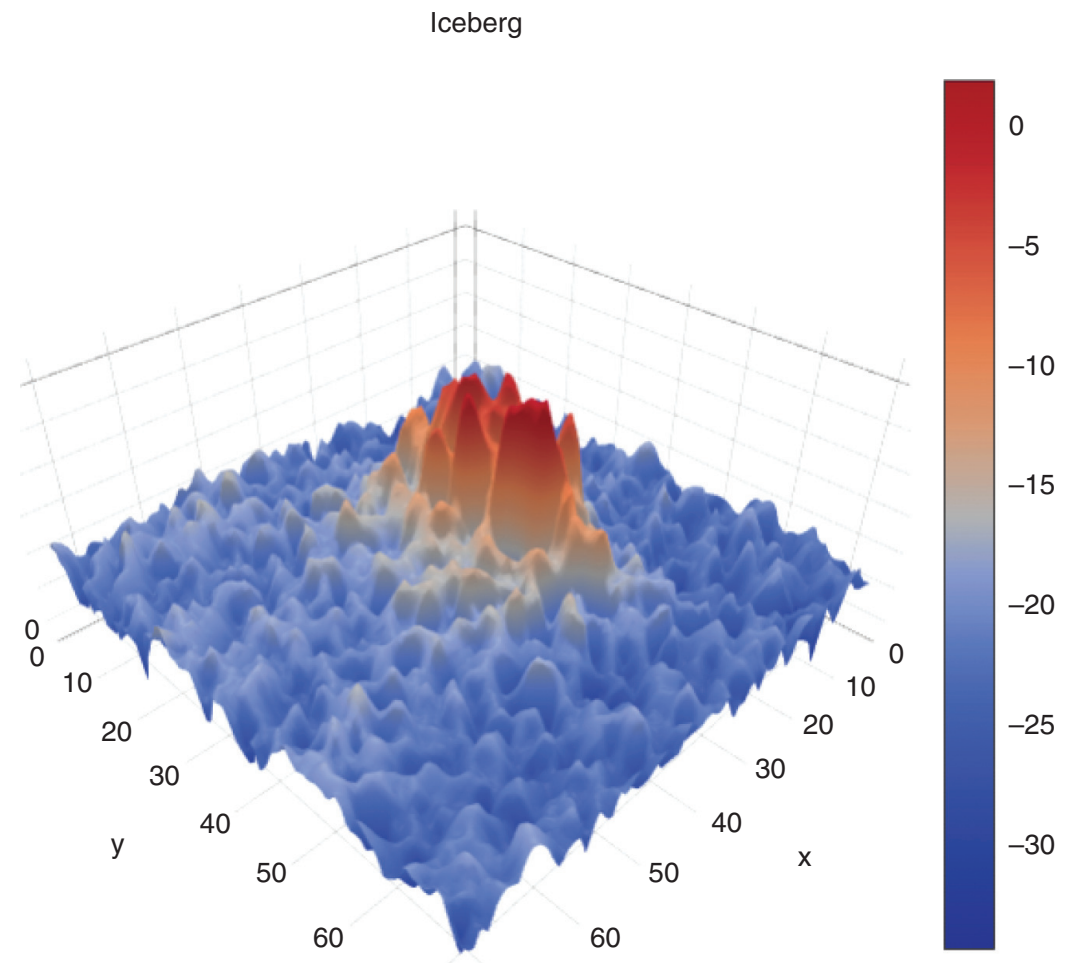

Export to plot.ly w

Figure 2: 3D Plot of a Iceberg. 
creating a third channel to get a three-channel RGB equivalent [3]. The 3D structure of ship and iceberg based on their respective band values is plotted using Plotly library is shown in Figures 1 and 2 respectively. As seen in the plots, the shape of the ship resembles an elongated point, whereas an iceberg has pinnacles and distortions. We exploit these structural differences to train the CNNs to learn the various features of ships and icebergs [1].

\section{Data Augmentation}

Data augmentation is a technique to add value to the base data that is information deprived by increasing the training data samples. It is common knowledge that the more data the CNN model has access to, the more will be its accuracy. By increasing the original dataset, data augmentation overcomes the overfitting of training samples. In this project, we make use of traditional transformations that manipulate the training data. Generally, the original images are either shifted, zoomed in/out, rotated, flipped, or distorted to get the augmented images. In this case, the images are augmented by flipping the image as this helps the CNN get a different orientation of the image. The flipped images, although a replica of their original images, help improve the training accuracy significantly. This is because the CNN being inherently invariant considers the flipped image almost as good as a new one and it may trigger the convolutional kernel matrix very differently compared to the original. Both image and its duplicate are fed into the neural network. For a dataset of size $N$, we generate a dataset of $3 N$ size (horizontal + vertical flips) as a result of data augmentation. After augmenting the data, the training set size increases to 4812 images. Thus, data augmentation is not only useful in classification tasks lacking sufficient amount of data but also helps improve the state-of-the-art algorithms for classification [7]. Augmenting the dataset increased the CNN training accuracy by approximately 1.2\%.

\section{Building the CNN Model}

CNN is a class of deep feed-forward artificial neural network that is extensively applied in the field of computer vision. CNN is a concept inspired by the structure and connectivity of the neurons in the human brain. With recent advancements in neural networks, CNNs have proven to be very effective in areas such as image recognition and classification. ConvNets have been successful in identifying faces, objects, and traffic signs apart from powering vision in robots and self-driving cars. In this project, we build a CNN comprising four main components: (1) Conv2D layer, (2) nonlinear activation function (ReLu), (3) pooling layer, and (4) dropout layer (to overcome overfitting). The model was built with a batch size of 32, which defines the number of samples propagated through the network per batch. The model comprises four Conv2D layers followed by three dense layers. The CNN also was implemented with a $3 \times 3$ convolutional filter. We use a 0.2 dropout layer and $2 \times 2$ maxpool layer after every convolutional layer [7]. The dropout layer is to reduce overfitting. Lastly, we had a fully connected layer consisting of 256 fully connected neurons. All convolutional layers used the "same" padding method and a "relu" activation function. The model consisted of a total of 560,193 parameters. A summary of the model can be seen in Figure 3.

\section{Semisupervised Approach: Pseudolabeling}

The most talked about and widely used ML techniques are supervised and unsupervised learning. However, there exists a third classification that incorporates the best of both supervised and unsupervised learning, which is known as semisupervised learning. Semisupervised learning is a class of supervised learning that makes use of both labeled and unlabeled data for training the model. This approach is typically adopted when there is a small amount of labeled data and large amount of unlabeled data. This technique has proven to be useful for a variety of reasons. First, manually labeling huge datasets for supervised learning can be very tedious and time consuming. Moreover, too much labeling can lead to human biases on the model. This can 


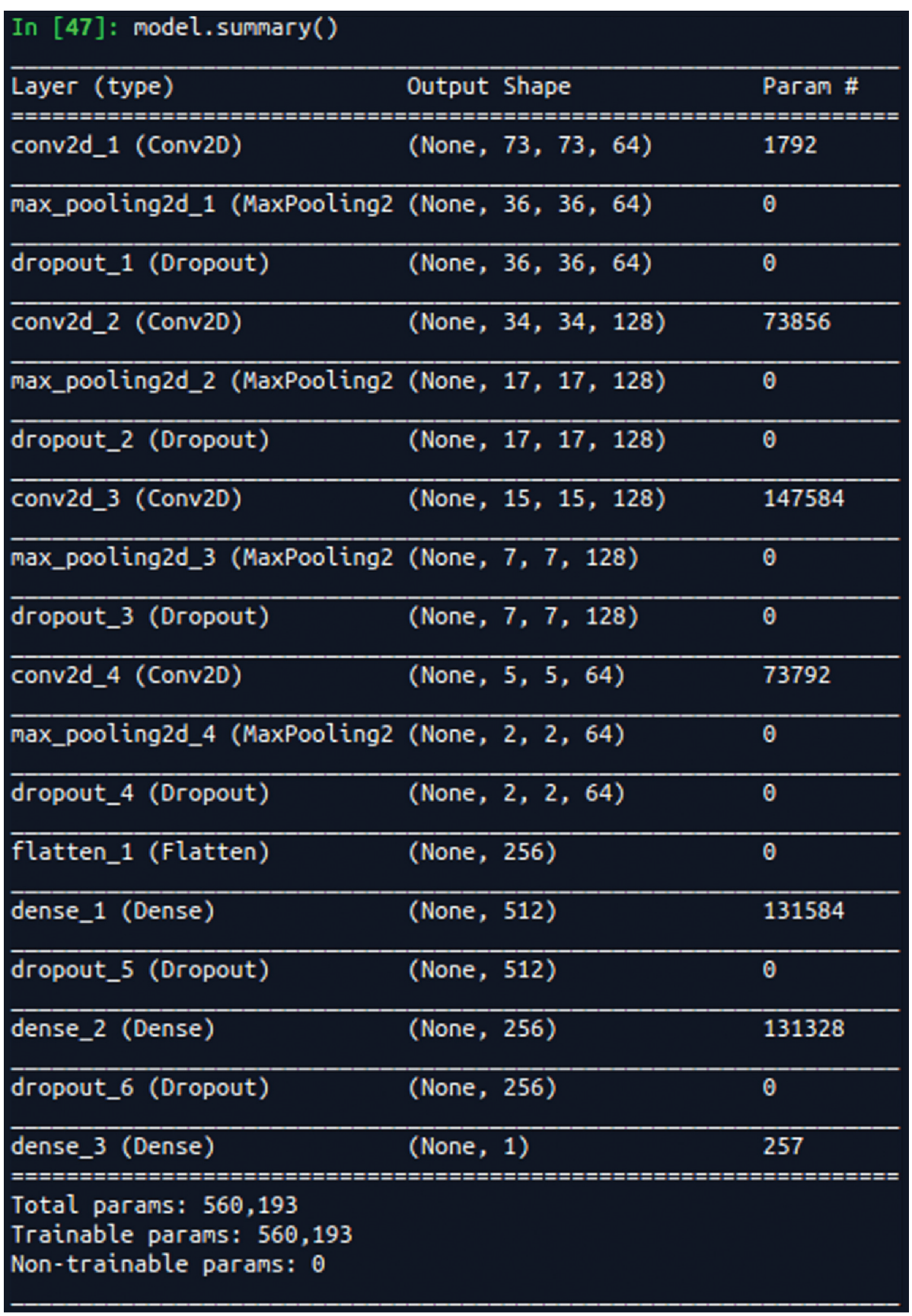

Figure 3: CNN Model Summary.

be overcome by including a lot of unlabeled data in training to increase the accuracy of the model. For this very reason, semisupervised learning is suitable for use cases such as webpage classification, speech recognition, or genetic sequencing. The lack of sufficient amount of training data is a challenging ML problem, but it is also a realistic one: in a lot of real-world use cases, even small-scale data collection can be extremely expensive or sometimes near impossible (e.g. in medical imaging). Being able to make the most out of very little data is a key skill of a competent data scientist [2].

Pseudolabeling is a simple and efficient method to do semisupervised learning. This technique is usually applied when a small chunk of data is labeled (training set) and a large set of test samples is unlabeled. This means that the model can only learn features from the small training set because it is labeled. The test data containing more valuable information cannot be used to our advantage as it is unlabeled. Therefore, this is 

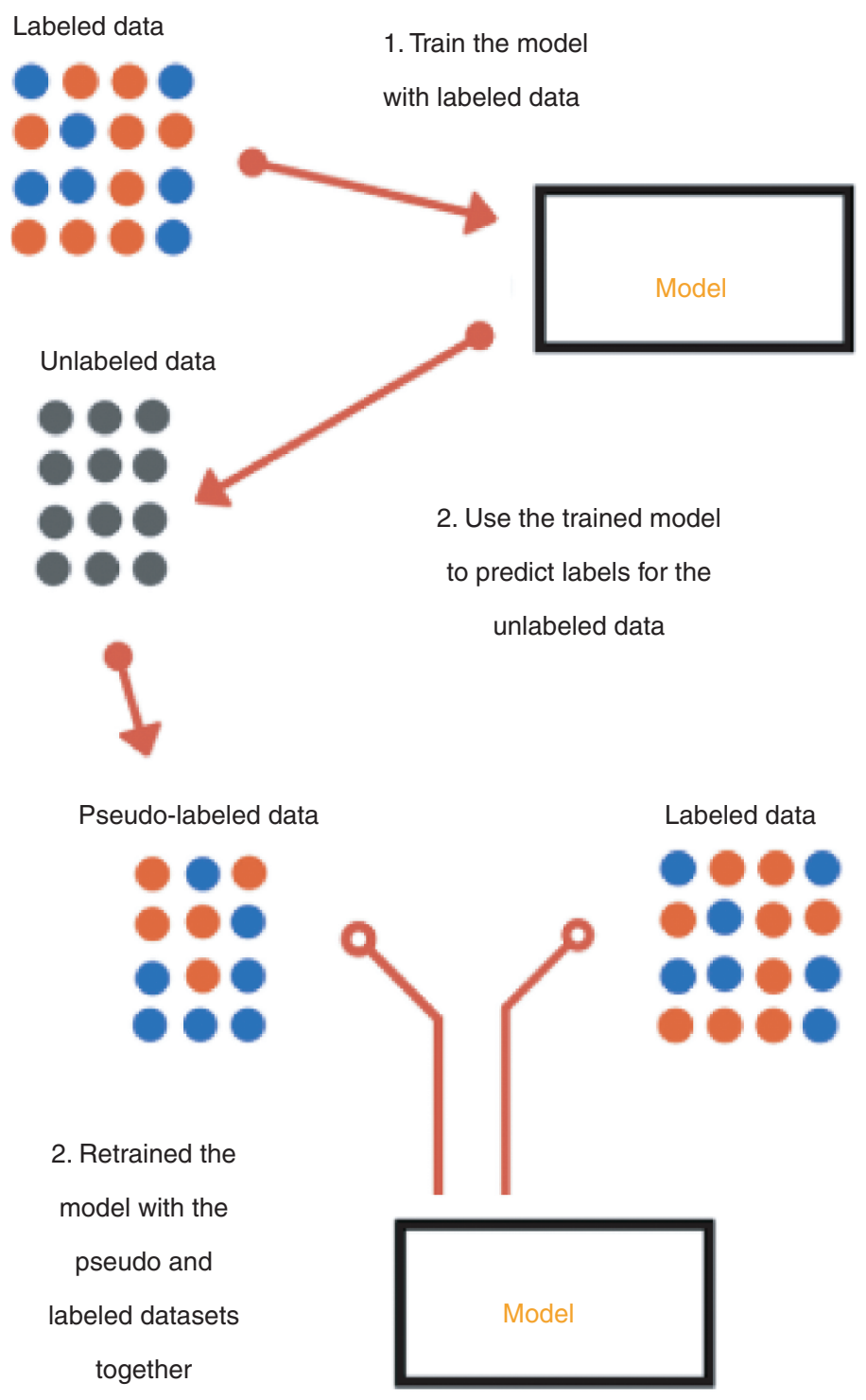

Figure 4: Pseudolabeled Model.

where pseudolabeling plays a vital role using the test data in model training. The diagram (Figure 4) and pseudocode shows the steps to follow for pseudolabeling.

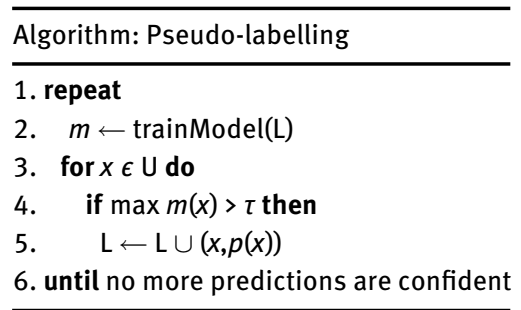

In this approach, pseudolabeling (self-training) trains a model $m$ on labeled training set $L$ and an unlabeled dataset $U$. With every iteration, the model $m$ predicts $m(x)$ in the form of a probability distribution over the $N$ classes for unlabeled example $x$ in $U$. If the predicted probability is higher than a threshold value $\tau$, 
then $x$ is concatenated to the labeled samples with $p(x)=\operatorname{argmax} m(x)$. This process is repeated until all the confident predictions in the unlabeled set are exhausted.

\section{Experiment and Evaluation}

The CNN structure is optimized by tuning the hyperparameters to attain optimal performance during model training. A number of convolutional layer sizes were tested, and the model giving the most optimal train accuracy was selected. Various callback functions were applied to the model while training it on 50 epochs. The CNN model was trained and tested on an augmented set using a stratified shuffle split to ensure that the folds are made by preserving the percentage of samples for each class. Figure 5 shows the CNN model accuracy on training it with the augmented data containing 4812 data samples. Once the CNN model is trained on augmented training set, we use this CNN to predict the probabilities of the test set samples being a ship or an iceberg. We now follow the semisupervised learning approach called pseudolabeling to make use of samples in the test set, which in turn will help us build a stronger CNN for classification. To incorporate the test samples, we only consider the samples that have a high probability of correct classification predicted by the CNN trained on augmented set (CNN model 1). We choose test samples to be labeled as "iceberg" whose predicted probability of being an iceberg by the CNN model 1 is greater than $95 \%$ and similarly choose samples to be labeled as "ship" whose probability of being an iceberg is lesser than 5\% [5]. On identifying these test samples, we concatenate them with the augmented data, thus increasing our training data that now contains 7372 samples.

The training set after concatenating test set pseudolabels is now to be trained on the CNN model using five-fold cross-validation. Various callbacks are applied while training the model on 30 epochs. Figure 6 shows the accuracy and scores of the enhanced CNN model containing the pseudolabeled data. The CNN model achieved validation accuracy of approximately $95 \%$ when tested and trained on five-fold cross-validation.

The enhanced CNN model (trained on pseudolabels) is tested on the test set and the output is the predicted probabilities of a sample being a ship or an iceberg. The sample IDs and their respective probabilities are written into a .csv file and submitted to Kaggle for the calculation of the LB score. We achieved an LB score of 0.214 with pseudolabeling as shown in Table 1 .

In this project, we further explored the semisupervised techniques of cotraining and generative modeling. The results of these approaches were then compared to that achieved by pseudolabeling (self-training). The basic idea of cotraining involves training two classifiers on two different views (features) of the data. Then, classifier 1's most confident predictions are added to classifier 2's training set and vice versa. Then, both classifiers retrain themselves with their respective datasets and in the process teach each other. The classifiers

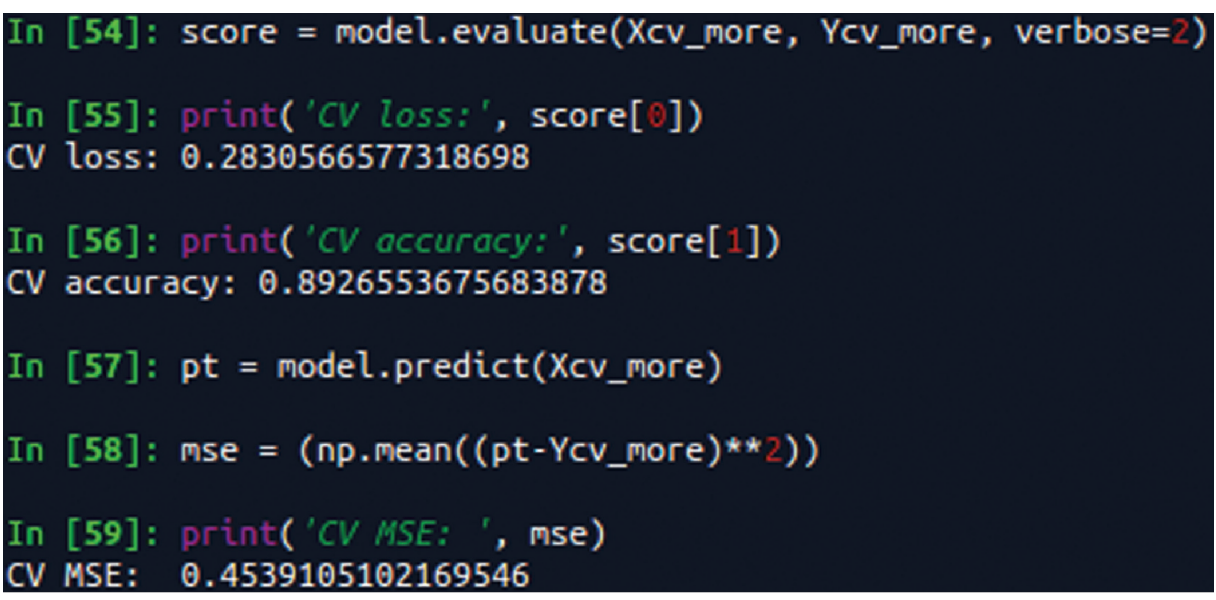

Figure 5: CNN Training Accuracies. 


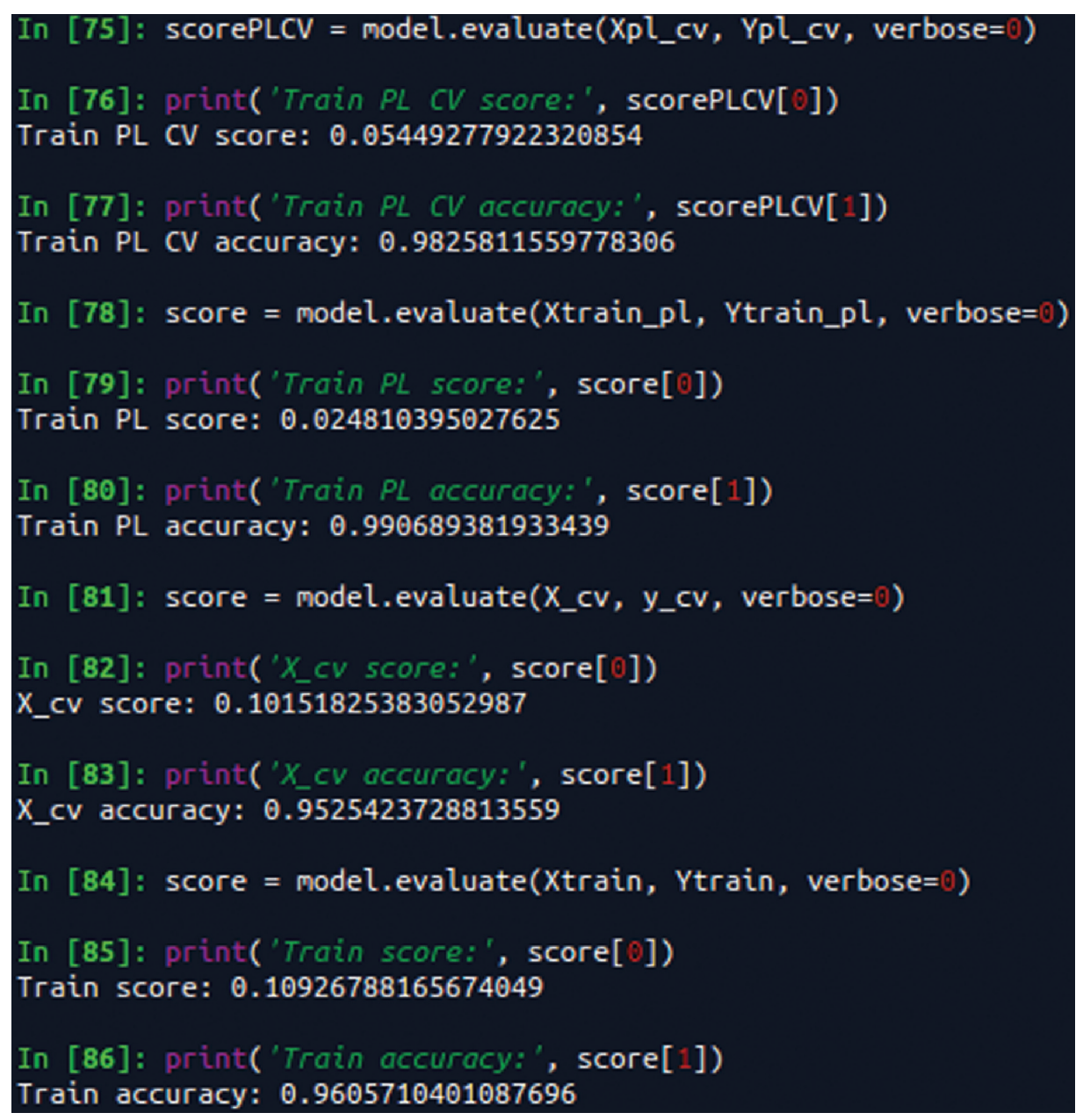

Figure 6: Pseudolabeled Training Accuracies.

Table 1: Semisupervised Learning Approaches Performance Measure.

\begin{tabular}{lr}
\hline Semisupervised approaches & LB score \\
\hline Pseudolabeling (self-learning) & 0.214 \\
Cotraining & 0.253 \\
Generative models & 0.317 \\
\hline
\end{tabular}

Table 2: Accuracy Measures on Validation set for Semisupervised Approaches.

\begin{tabular}{|c|c|c|c|c|}
\hline Semisupervised approaches & Precision & Recall & Sensitivity & Specificity \\
\hline Pseudolabeling (self-learning) & $95.2 \%$ & $94.7 \%$ & $95.1 \%$ & $95.3 \%$ \\
\hline Cotraining & $91.7 \%$ & $90.1 \%$ & $91.3 \%$ & $92.2 \%$ \\
\hline Generative models & $85.4 \%$ & $85 \%$ & $85.7 \%$ & $86.1 \%$ \\
\hline
\end{tabular}

built using cotraining achieved validation accuracy of approximately $91 \%$ using five-fold cross-validation. On evaluating the predictions by the cotrained model, we received an LB score of 0.253 (a lower score implies better classification accuracy as shown in Table 1). One of the setbacks of this model was that it was computationally time-consuming, and given that we were dealing with limited data features, it was difficult to split these features into two. Second, we tried generative modeling for the classification task. To score the predictions made by the generative model, we used five-fold cross-validation, which resulted in an accuracy of approximately $85 \%$ as shown in Table 2 . The predictions resulted in an LB score of 0.317 . The classification 
accuracy declined further because generative models find it difficult to balance the impact of labeled and unlabeled data when the unlabeled data outnumbers the labeled data. Finally comparing the scores of these models as shown below, pseudolabeling gave us the best results in terms of LB score and computation time.

\section{Conclusion}

This paper presents the use of CNNs for ship-iceberg discrimination in high-resolution SAR images. The main challenge in this project was that the size of test data (unlabeled) was much larger than the train data (labeled). To overcome this constraint, we resorted to pseudolabeling, which is a semisupervised ML approach. We also compared the LB score of the CNN model predictions with and without pseudolabeling. It was observed that the LB score improved from 0.4515 to 0.214 with pseudolabeling, beating more than half of the competing teams. Our work can be further improved by exploring the preprocessing of our data. In our analysis, we disregarded the incidence angle that may have vastly influenced our result. We are aware that incidence angle can affect the intensity of the satellite decibel readings and we believe that the integration of this parameter is necessary to push the classification accuracy further. Given the log-loss scoring method used in this competition, classification error can have an exponential impact on submission scores. Thus, in our future work, we hope to reduce our classification error through various alternative data preprocessing methods and the exploration of different CNN architecture and hyperparameters.

\section{Bibliography}

[1] C. Bentes, A. Frost, D. Velotto and B. Tings, Ship-iceberg discrimination with convolutional neural networks in high resolution SAR images, in: Processing of EUSAR 2016: 11th European Conference on Synthetic Aperture Radar.

[2] F. Chollet, Building powerful image classification models using very little data.

[3] H. Jang, S. Kim and T. Lam, Kaggle competitions: author identification and Statoil/C-CORE Iceberg Classifier Challenge, School of Informatics, Computing, and Engineering, Indiana University, Bloomington, IN, USA, Datamining B565 Fall 2017.

[4] L. Jun, P. Atharva and S. Dhruv, Iceberg-ship classifier using SAR image maps, Final Project Report 2017, Stanford University.

[5] D.-H. Lee, Pseudo-label: the simple and efficient semi-supervised learning method for deep neural networks, in: ICML 2013 Workshop: Challenges in Representation Learning (WREPL), Atlanta, GA, USA, 2013.

[6] J. Tang, C. Deng, G.-B. Huang and B. Zhao, Compressed-domain ship detection on spaceborne optical image using deep neural network and extreme learning machine, IEEE Trans. Geosci. Remote Sens. 53 (2015), 1174-1185.

[7] P. Velickovic, Deep learning for complete beginners: convolutional neural networks with keras.

[8] G. Wu, J. de Leeuw, A. K. Skidmore, Y. Liu and H. H. T. Prins, Performance of Landsat TM in ship detection in turbid waters, Int. J. Appl. Earth Obs. Geoinf. 11 (2009), 54-61. 